\title{
Compact green-diode-based lasers for biophotonic bioimaging
}

Jensen, Ole Bjarlin; Hansen, Anders Kragh; Petersen, Paul Michael; Müller, André; Sumpf, Bernd; Unterhuber, Angelika; Drexler, Wolfgang; Andersen, Peter E.

\section{Published in:}

SPIE Newsroom

Link to article, DOI:

10.1117/2.1201401.005287

Publication date:

2014

Document Version

Publisher's PDF, also known as Version of record

Link back to DTU Orbit

Citation (APA):

Jensen, O. B., Hansen, A. K., Petersen, P. M., Müller, A., Sumpf, B., Unterhuber, A., Drexler, W., \& Andersen, P. E. (2014). Compact green-diode-based lasers for biophotonic bioimaging. SPIE Newsroom. https://doi.org/10.1117/2.1201401.005287

\section{General rights}

Copyright and moral rights for the publications made accessible in the public portal are retained by the authors and/or other copyright owners and it is a condition of accessing publications that users recognise and abide by the legal requirements associated with these rights.

- Users may download and print one copy of any publication from the public portal for the purpose of private study or research.

- You may not further distribute the material or use it for any profit-making activity or commercial gain

- You may freely distribute the URL identifying the publication in the public portal 


\section{Compact green-diode-based lasers for biophotonic bioimaging}

Ole Bjarlin Jensen, Anders Kragh Hansen, Paul Michael

Petersen, André Müller, Bernd Sumpf, Angelika Unterhuber, Wolfgang Drexler, and Peter E. Andersen

Diode lasers simultaneously offer tunability, high-power emission, and compact size at fairly low cost and are increasingly preferred for pumping titanium:sapphire lasers.

Lasers based on semiconductor materials, so-called diode lasers, are highly efficient, compact light sources. ${ }^{1}$ In high volume they can also be produced at very low cost. In contrast to lasers based on specific atomic transitions, such as solid-state or gas lasers, diode laser materials engineering enables generation of light in a large portion of the optical spectrum. ${ }^{2}$ The great versatility of diode lasers allows their direct use in many biophotonics applications, including photocoagulation, ${ }^{3}$ diffuse optical imaging, ${ }^{4}$ and optical coherence tomography (OCT). .6

Nonlinear frequency conversion of diode lasers is an attractive method of overcoming the limitations of solid-state lasers. ${ }^{1}$ However, efficient frequency conversion requires optimizing parameters such as spectral and spatial quality. With the introduction of high-brightness tapered diode lasers, beam quality has reached a level suitable for direct high-power frequency conversion. Frequency doubling of these lasers in periodically poled crystals has resulted in up to $2 \mathrm{~W}$ of output power in the green spectral range. 7,8 Moreover, combining two of these lasers with subsequent sum-frequency generation provides close to $4 \mathrm{~W}$ of nearly diffraction-limited green light ${ }^{9}$ without the need for external cavities. Opportunities also exist for further increasing green output power. ${ }^{10}$ Importantly, these power levels meet the requirements for direct application of lasers in treatment of vascular diseases and for pumping of titanium (Ti):sapphire lasers. ${ }^{11}$

In the diagnosis of disease, the broad spectrum and ultrashort pulses of Ti:sapphire lasers help to increase the resolution of different imaging techniques, for example, multiphoton
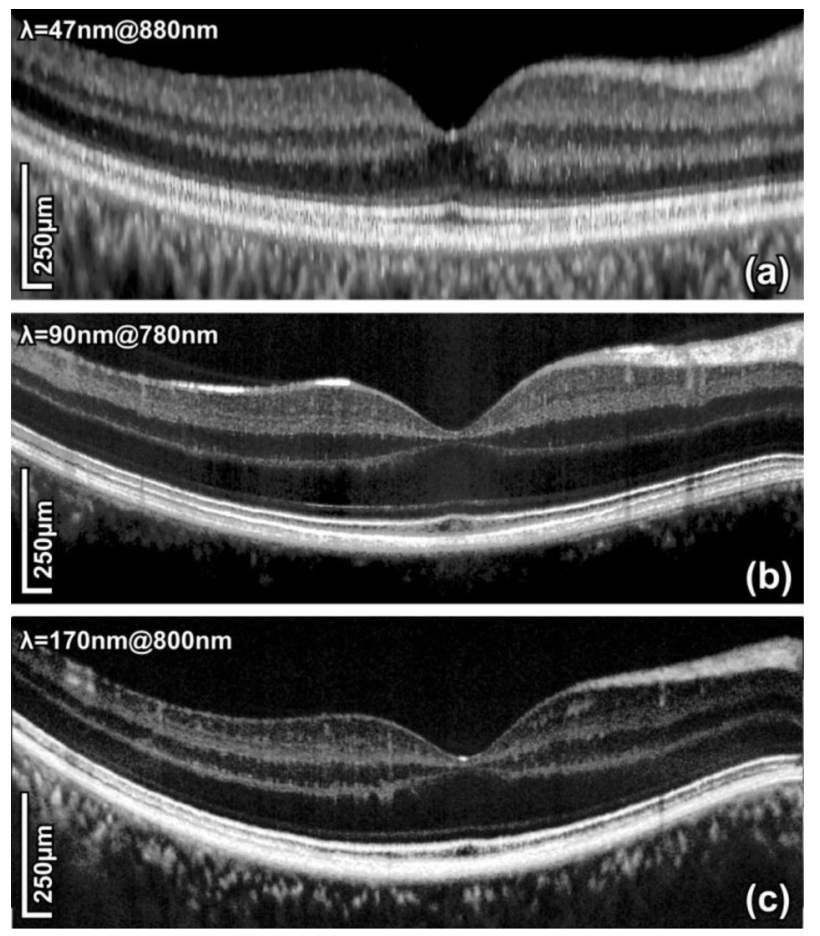

Figure 1. Tomograms using the retracking function of the tapered laser diode device with (a) an internal light source (100 frames) compared with (b) the frequency-doubled tapered diode laser (50 frames) and (c) Femtolasers' Integral Core, a commercial titanium (Ti):sapphire laser (20 frames). Tomograms are displayed in a 1:3 ratio (horizontal:vertical) for better visualization of the layered structure. $\lambda$ : Bandwidth (full width at half-maximum) at specified center wavelength. (From Unterhuber et al., ${ }^{13}$ 2013.)

tomography $^{12}$ and OCT. ${ }^{5}$ Ti:sapphire lasers are pumped in the green spectral range, and traditionally, frequency-doubled diode-pumped solid-state lasers (DPSSs) are the light sources 
preferred due to their high output power and excellent beam properties. However, compact and efficient pump sources might enable further widespread adoption of Ti:sapphire lasers, in particular within multimodal bioimaging applications.

OCT is a well-established imaging technique that enables depth-resolved images with high resolution. 5,6 The large bandwidth of Ti:sapphire lasers is attractive for ultra-high-resolution OCT imaging. By the same token, the main limitation of Ti:sapphire lasers in OCT systems is their relatively high cost, which is mainly determined by the DPSS pump sources used. A shift of pump sources to frequency-doubled diode lasers may reduce the cost of the devices and provide access to more compact Ti:sapphire lasers in the future, with all of their advantages.

Here we describe initial results of in vivo OCT imaging of the retina and skin using a Ti:sapphire laser pumped by a frequency-doubled, distributed Bragg reflected (DBR) tapered diode laser. ${ }^{13}$ For the ophthalmic imaging, we used a modified Spectralis OCT device (Heidelberg Engineering) with a built-in fixation target and eye-tracking device. The system was modified to permit use of wide-bandwidth light sources at 800 and 1060nm. We modified the Ti:sapphire laser to provide stable mode-locked operation with an output power of $80 \mathrm{~mW}$ at $1.4 \mathrm{~W}$ pump power with a spectral width of 90nm (200nm full width at half-maximum), which provides an axial resolution of $<4 \mu \mathrm{m}$. We coupled the light from the Ti:sapphire laser to an optical fiber and regulated the output power from the fiber to give a maximum of $0.8 \mathrm{~mW}$ at the cornea, well below safety limits. Figure 1(b) shows the resulting image, along with comparisons with the built-in source-Figure 1(a)-and a commercial Ti:sapphire laser-Figure 1(c).

The use of a broad-bandwidth laser source enabled despeckling at high axial resolution and visualization of detailed retinal anatomy, such as the major retinal layers and the fine structure of

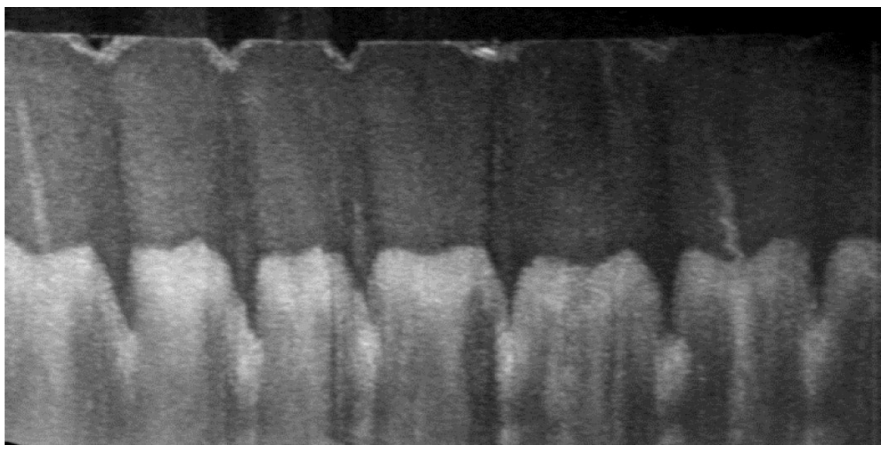

Figure 2. Optical coherence tomography image of skin using a Ti:sapphire laser pumped by a frequency-doubled tapered diode laser. the retinal pigment epithelium-choroid complex, as well as that of the optic nerve, including the lamina cribrosa, where identifying structural changes is crucial in diagnosing glaucoma. ${ }^{10,13}$

In a home-built OCT system tailored for in vivo skin imaging, we used the Ti:sapphire laser to demonstrate high-resolution imaging of skin. Figure 2 shows a tomogram of the left index finger of a healthy subject with penetration depth of about $800 \mu \mathrm{m}$, clearly visualizing subsurface structures of normal skin, including the epidermis, dermoepidermal junction, dermis, and sweat ducts. ${ }^{10}$

In summary, frequency-converted tapered diode lasers show great potential for many applications in biophotonics. In particular, the high axial resolution and good contrast of OCT images clearly show the potential of using Ti:sapphire lasers pumped by frequency-doubled DBR tapered diode lasers for biophotonic imaging. The lower cost and footprint of these pump lasers should enable significant widespread adoption in diagnostics, for example, OCT and multiphoton imaging. Our aim, and a work in progress, is to combine several such imaging modalities on a common platform driven by one light source, thus enabling compact, multimodal imaging.

The authors acknowledge financial support from the European Union project FAMOS (Seventh Framework Programme, Information and Communication Technologies, contract 317744).

\section{Author Information}

\section{Ole Bjarlin Jensen, Anders Kragh Hansen, Paul Michael}

Petersen, and Peter E. Andersen

DTU Fotonik

Technical University of Denmark

Roskilde, Denmark

Ole Bjarlin Jensen is a senior researcher in the Department of Photonics Engineering at DTU Fotonik. For more than 10 years he has worked on diode lasers, solid-state lasers, and nonlinear frequency conversion, as well as biomedical applications of laser systems.

Anders Kragh Hansen joined DTU Fotonik as a postdoctoral fellow in 2013. He is currently working on research and development of laser modules involving nonlinear frequency conversion with laser diodes as sources. Biophotonics applications are of special importance to this work. 
Paul Michael Petersen is a professor. He has more than 25 years of research experience in laser physics, nonlinear optics, and optical measuring techniques. He has headed several collaborative research projects within laser physics and LED lighting.

Peter E. Andersen, $\mathrm{PhD}$, is a senior scientist and research professor at the Technical University of Denmark, where he leads research in biomedical optics. He has more than 15 years of research experience in light sources for biomedical optics, bioimaging, and OCT systems.

\section{André Müller and Bernd Sumpf}

Ferdinand-Braun-Institut

Leibniz-Institut für Höchstfrequenztechnik

Berlin, Germany

André Müller joined the Ferdinand-Braun-Institut in 2013. He works on high-brightness diode lasers and diode laser systems for spectroscopic applications.

Bernd Sumpf has 20 years of experience in developing lasers. He has been at the Ferdinand-Braun-Institut since 2000. His current research topics are high-brightness diode lasers and diode lasers for sensory applications and Raman spectroscopy.

\section{Angelika Unterhuber and Wolfgang Drexler}

Center for Medical Physics and Biomedical Engineering

Medical University of Vienna

Vienna, Austria

Angelika Unterhuber has 15 years of experience in ultrafast optics. Currently, she works at the Medical University of Vienna where her research interests are focused on development of novel light sources and their biomedical application, especially in the field of OCT and nonlinear imaging.

Wolfgang Drexler has more than 20 years of experience in biophotonic imaging and OCT. Since 2009 he has been full professor of medical physics and head of the Center for Medical Physics and Biomedical Engineering. He is a member of the Austrian Academy of Sciences and has more than 150 publications.

\section{References}

1. A. Müller, S. Marschall, O. B. Jensen, J. Fricke, H. Wenzel, B. Sumpf, and P. E. Andersen, Diode laser based light sources for biomedical applications, Laser Photonics Rev. 7, pp. 605-627, 2013.

2. P. Unger, Introduction to power diode lasers, in R. Diehl ed., High Power Diode Lasers: Fundamentals, Technology, Applications, pp. 1-54, Springer, Berlin, 2000 3. K. Suthamjariya and R. R. Anderson, Lasers in dermatology, in T. Vo-Dinh ed., Biomedical Photonics Handbook, pp. 40-1 to 40-28, CRC Press, Boca Raton, FL, 2003.

4. L. V. Wang and H.-i. Wu, Biomedical Optics: Principles and Imaging, Wiley, 2007.

5. W. Drexler and J. G. Fujimoto, Optical Coherence Tomography: Technology and Applications, Springer, 2008.

6. S. Marschall, B. Sander, M. Mogensen, T. M. Jørgensen, and P. E. Andersen, Optical coherence tomography — current technology and applications in clinical and biomedical research, Anal. Bioanal. Chem. 400 (9), pp. 2699-2720, 2011.

7. O. B. Jensen, P. E. Andersen, B. Sumpf, K.-H. Hasler, G. Erbert, and P. M. Petersen, $1.5 \mathrm{~W}$ green light generation by single-pass second harmonic generation of a singlefrequency tapered diode laser, Opt. Express 17, pp. 6532-6539, 2009.

8. D. Jedrzejczyk, R. Güther, K. Paschke, W.-J. Jeong, H.-Y. Lee, and G. Erbert, Efficient high-power frequency doubling of distributed Bragg reflector tapered laser radiation in a periodically poled $\mathrm{MgO}$-doped lithium niobate planar waveguide, Opt. Lett 36, pp. 367369, 2011.

9. A. Müller, O. B. Jensen, K.-H. Hasler, B. Sumpf, G. Erbert, P. E. Andersen, and P. M. Petersen, Efficient concept for generation of diffraction-limited green light by sumfrequency generation of spectrally combined tapered diode lasers, Opt. Lett 37, pp. 37533755, 2012.

10. O. B. Jensen, A. K. Hansen, A. Müller, B. Sumpf, A. Unterhuber, W. Drexler, P. M. Petersen, and P. E. Andersen, Power scaling of nonlinear frequency converted diode lasers for biophotonics, IEEE J. Sel. Topics Quantum Electron. 20, p. 7100515, 2013. doi:10.1109/JSTQE.2013.2284423

11. A. Müller, O. B. Jensen, A. Unterhuber, T. Le, A. Stingl, K.-H. Hasler, B. Sumpf, G. Erbert, P. E. Andersen, and P. M. Petersen, Frequency-doubled DBR-tapered diode laser for direct pumping of Ti:sapphire lasers generating sub-20 fs pulses, Opt. Express 19, pp. 12156-12163, 2011.

12. E. E. Hoover and J. A. Squier, Advances in multiphoton microscopy technology, Nat. Photonics 7, pp. 93-101, 2013

13. A. Unterhuber, B. Považay, A. Müller, O. B. Jensen, M. Duelk, T. Le, P. M. Petersen, et al., Simultaneous dual wavelength eye-tracked ultrahigh resolution retinal and choroidal optical coherence tomography, Opt. Lett 38, pp. 4312-4315, 2013. 